Reprod. Nutr. Dévelop., 1982, 22 (4), 661-680.

\title{
Les stades de l'organogenèse et la cytodifférenciation de la glande à albumen de l'escargot Helix aspersa Müller
}

\author{
Anne-Marie COURTOT, L. GOMOT
}

Laboratoire de Zoologie et Embryologie, L. A. CNRS n ${ }^{\circ} 040310$,

Faculté des Sciences, Place Maréchal-Leclerc, 25030 Besançon Cedex, France.

Summary. Snail albumen gland cytodifferentiation during organogenesis.

The differentiation of the albumen gland of the pulmonate stylommatophora Helix aspersa has been divided into five stages. An ultrastructural study showed the differentiation of undifferentiated epithelial cells into two cell types : ciliated cells and secretory cells.

The glandular differentiation of epithelial cells was characterized by the development of ergastoplasma and the Golgi apparatus which were both involved in protein and galactogen synthesis.

\section{Introduction.}

La glande à albumen est une glande annexe de l'appareil génital des Mollusques Gastéropodes. Chez les Pulmonés, où elle est bien individualisée, en particulier chez Helix aspersa Müller, c'est une glande volumineuse, située à la jonction du canal hermaphrodite et du spermoviducte au niveau de la poche de fécondation. C'est une glande exocrine, tubulo-acineuse, à activité cyclique : elle produit du glycogène en hiver, du galactogène et des protéines en été. Ces deux dernières substances interviennent au moment de la ponte et constituent la couche périvitelline des œufs (Goudsmit, 1975).

Les recherches antérieures réalisées sur la glande à albumen sont relativement hétérogènes. On peut distinguer celles qui portent sur les Pulmonés adultes et celles qui s'adressent à des animaux en développement.

Chez les Pulmonés adultes, des travaux histochimiques et biochimiques ont permis d'analyser les mécanismes de la formation du galactogène (Goudsmit, 1964 ; Goudsmit et Ashwell, 1965 ; Goudsmit et Neufeld, 1967 ; Sawicka, 1967 ; Meenakshi et Scheer, 1969 ; Bolognani Fantin et Gervaso, 1971 ; Goudsmit et Friedman, 1976). Les études ultrastructurales sont peu nombreuses, cependant on dispose de la description des cellules de la glande à albumen d'Helix pomatia (Nieland et Goudsmit, 1969) et de Biomphalaria glabrata (De Jong-Brink, 1969).

Chez les jeunes Pulmonés, on possède des données intéressantes, mais uniquement chez les Limaces. Abeloos (1943) et Laviolette (1954) ont montré le rôle 
important de la gonade dans le développement de cet organe. Smith (1966) et Runham et Laryea (1968) ont précisé l'évolution de la glande à albumen au cours de la maturation du tractus génital.

Aussi, dans le cadre de nos recherches sur la différenciation sexuelle de I'Escargot, il nous a paru nécessaire d'étudier les étapes essentielles de l'organogenèse et de la cytodifférenciation de la glande à albumen d'Helix aspersa en microscopie électronique, en nous basant sur la détermination préalable des stades de développement en microscopie photonique.

\section{Matériel et Méthodes.}

\section{Animaux.}

Nous avons utilisé, pour nos expériences, de jeunes Escargots de l'espèce Helix aspersa élevés au laboratoire. Ces animaux proviennent de pontes recueillies par nos soins dans notre élevage. Après l'éclosion, les jeunes sont placés par lots homogènes dans des boîtes d'élevage en matière plastique translucide, et soumis aux conditions naturelles d'éclairement. La nourriture, humidifiée, distribuée deux fois par semaine, était constituée de laitue. La croissance de ces animaux est appréciée par mesure de leur plus grand diamètre. Des séries de 5 Escargots sont sacrifiés à intervalles réguliers tous les mois de l'âge de 1 à 6 mois.

\section{Techniques}

1) Cytochimie des acides nucléiques. - Après fixation au Carnoy et inclusion à la paraffine, les acides nucléiques ont été mis en évidence par le test de Brachet (1944) au vert de méthyl-pyronine selon la technique de Lison (1960).

2) Techniques de fixation et d'inclusion dans les résines. - Les glandes prélevées aux différents stades étudiés sont placées pendant $1 \mathrm{~h} 30$ dans le fixateur suivant : Glutaraldéhyde à $200 \mathrm{mOsM}, 1$ vol., Tampon cacodylate à $200 \mathrm{mOsM}$, 1 vol., $\mathrm{NaCl}$ à $350 \mathrm{mOsM}, 1$ vol.

Les organes sont ensuite rincés dans le mélange : Tampon cacodylate 200 mOsM, 1 vol., Solution $\mathrm{NaCl} 300$ mOsM, 1 vol.

Ce lavage est suivi d'une post-fixation à l'acide osmique ajusté à la pression osmotique de $250 \mathrm{mOsM}$ à l'aide de tampon cacodylate.

La déshydratation est faite selon la technique de Luft (1961) et les pièces sont incluses dans l'Epon 812 ou dans l'ERL 4206 selon Spürr (1969).

3) Méthodes de coloration.

a) Coloration des coupes semi-fines. Les coupes ont été colorées soit au bleu de toluidine selon la méthode de Trump et al. (1961), soit à I'APS, en ayant soin d'éliminer préalablement l'osmium du fixateur par un traitement à l'eau oxygénée, soit au Noir Soudan B selon la technique de McGee-Russel et Smale (1963).

b) Coloration des coupes ultrafines. Les coupes ultrafines recueillies sur grilles en cuivre sont contrastées à l'acétate d'uranyle à 3 p. 100 dans l'alcool éthylique à 50 p. 100, puis au citrate de plomb d'après Reynolds (1963). 
La mise en évidence des polysaccharides a été faite sur coupes récoltées sur grilles en or par la technique à l'acide périodique-thiocarbohydrazide-protéinate d'argent (PATAG) d'après Seligman, modifiée par Thiéry et Rambourg (1974) pour la mise en évidence du glycogène. Des contrôles ont été obtenus en remplaçant le bain de thiocarbohydrazide (TCH) par un bain d'acide acétique à 20 p. 100 .

\section{Résultats.}

Le tableau I résume les étapes de développement de la glande placées dans le contexte de l'organogenèse du tractus génital au cours des deux premières phases de croissance définies par Gomot et Enée (1980) jusqu'à la puberté.

Dans la chronologie d'apparition des organes de l'appareil reproducteur, la glande à albumen est présente chez les animaux âgés d'un mois sous forme d'une petite hernie à la jonction du canal hermaphrodite et du spermoviducte en cours de différenciation, tandis que l'ébauche des glandes multifides devient seulement visible au $3^{e}$ mois.

\section{TABLEAU 1}

Principales phases de l'organogenèse génitale d'Escargots élevés en laboratoire

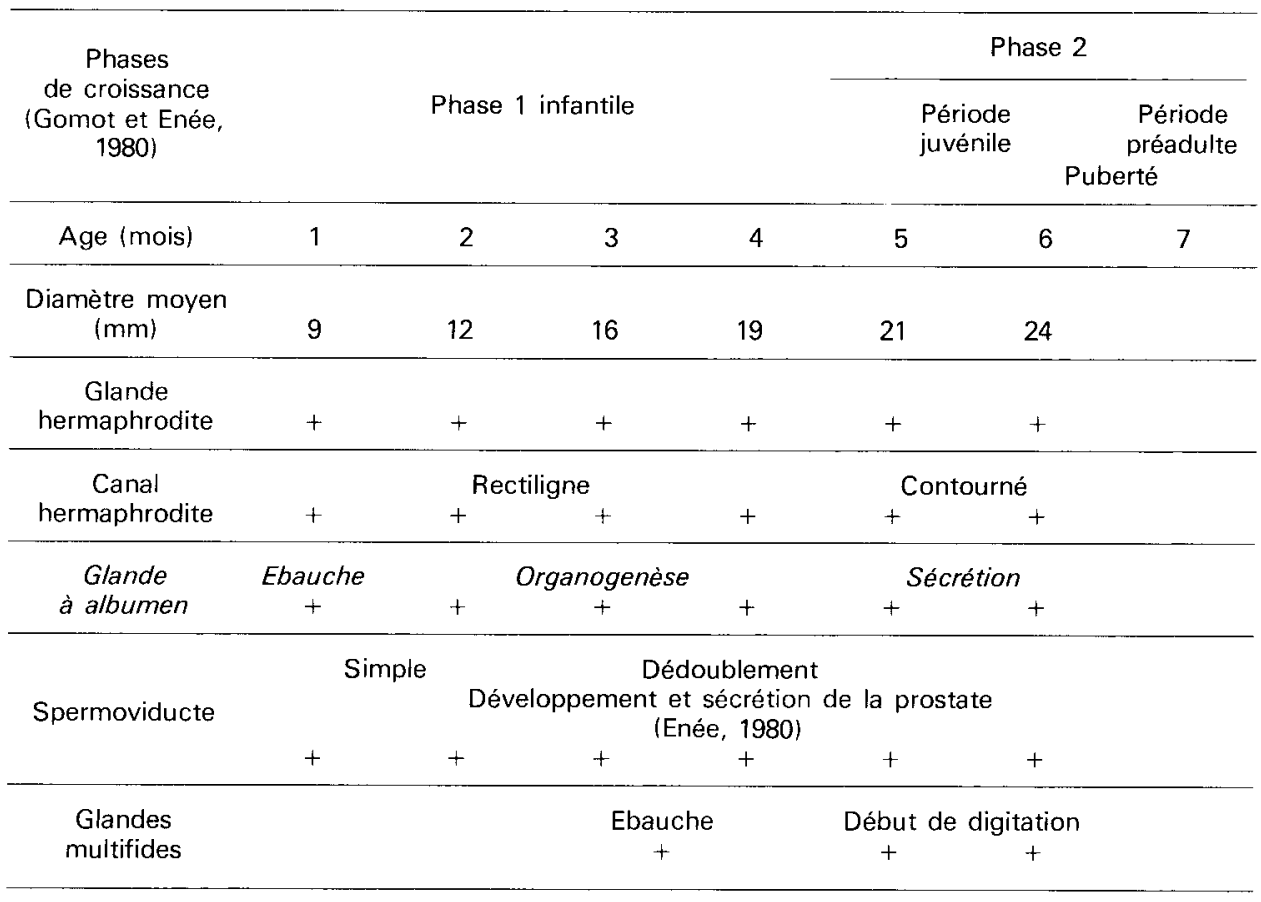

+ : organe visible macroscopiquement. 


\section{Définition des stades de l'organogenèse par examen en microscopie photonique.}

Etant donné qu'il existe une variabilité importante (en cours d'étude) du diamètre des animaux issus d'une même ponte, nous avons subdivisé l'évolution de la glande en fonction de son aspect histologique, tout en donnant l'âge et le diamètre de I'animal. Cinq stades ont ainsi été déterminés :

- Stade 1 : tubulaire simple (fig. 1). La glande se présente sous forme d'un tube circonvolué dont la paroi est constituée d'un épithélium pseudostratifié. On observe cet état chez des Escargots infantiles de 2 mois et de $13 \mathrm{~mm}$ de diamètre.

- Stade 2: tubulaire composé (Escargots infantiles de 4 mois et de $20 \mathrm{~mm}$ de diamètre). Le tube simple émet des bourgeonnements qui sont à l'origine des tubules primaires de la glande (fig. 2). L'axe mitotique des cellules est parallèle à la lumière du tube et la division des cellules provoque une élongation de ce dernier. A ce stade, le test de Brachet révèle la présence de nombreux ribosomes localisés principalement à l'apex des tubes.

- Stade 3 : formation des acini (Escargots juvéniles de 5 mois et de $21 \mathrm{~mm}$ de diamètre). Les acini se forment par ramification des tubes primaires. La glande acquiert sa structure caractéristique (fig. 3). Cette étape précède l'apparition de la sécrétion. Le tissu mésenchymateux s'organise en enveloppe de la glande et des acini qu'il entoure de façon intime au fur et à mesure du bourgeonnement et de la dichotomisation des tubes sécréteurs.

- Stade 4 : apparition des premiers grains de sécrétion (Escargots juvéniles de 5 mois et de $22 \mathrm{~mm}$ de diamètre). C'est une phase de transition entre la fin de la formation des acini et l'entrée en fonction de la glande. Tandis que certaines cellules se multiplient encore, d'autres présentent des signes de sécrétion. Les deux catégories de cellules sont réparties de façon homogène dans la glande (fig. 4). - Stade 5 : sécrétoire (Escargots de 6 mois - puberté - et de $25 \mathrm{~mm}$ de diamètre). On observe alors deux types cellulaires dans les acini (fig. 5) :

- des cellules sécrétrices, à polarité nette ; les grains de sécrétion sont apicaux et le noyau, volumineux, est rejeté à la partie basale de la cellule :

- des cellules non sécrétrices, intercalées entre les cellules sécrétrices ; leur noyau allongé prend progressivement une position apicale et ces cellules deviennent des cellules ciliées.

FIG. 1. - Aspect en coupe transversale de l'ébauche de la glande à albumen au stade tubulaire simple. lu : lumière de l'ébauche tubulaire; tc : tissu conjonctif.

FIG. 2. - Coupe transversale de la glande à albumen au stade tubulaire composé. Les tubes primaires

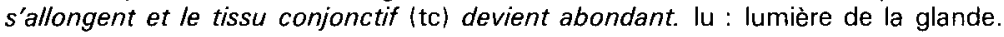

FIG. 3. - Zone centrale de la glande à albumen après ramification des tubes primaires et formation de nombreux acini. 
(1)

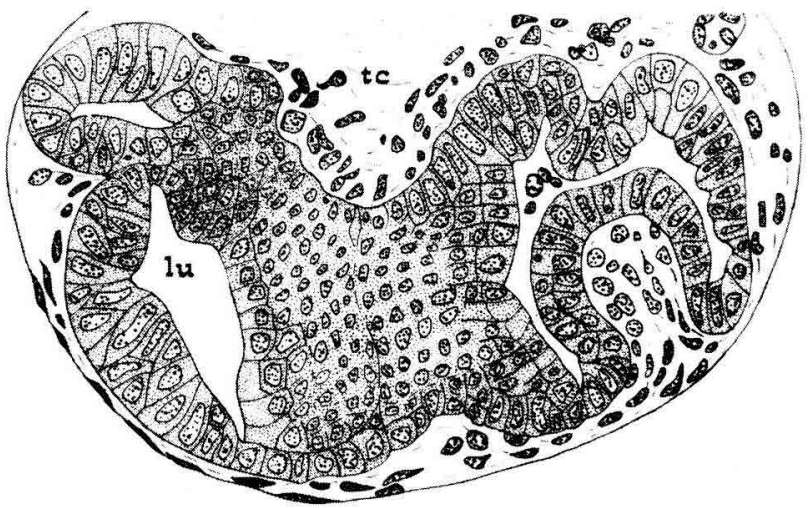

(2)

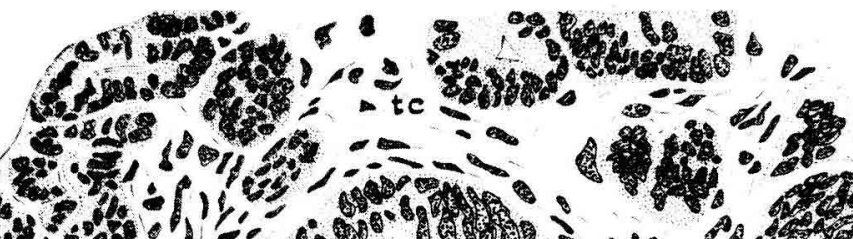
nitan

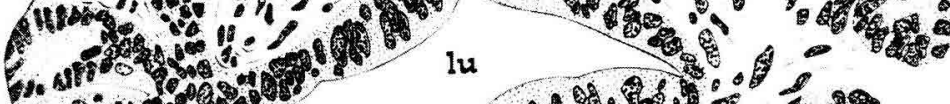

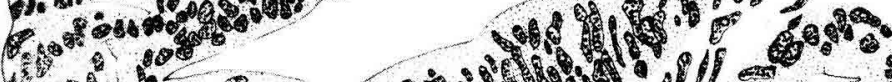

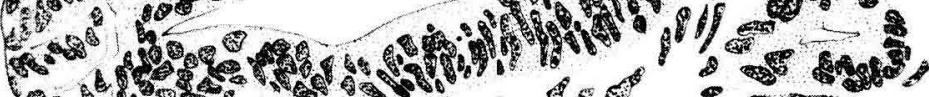

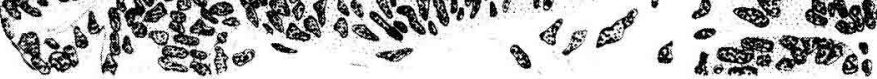

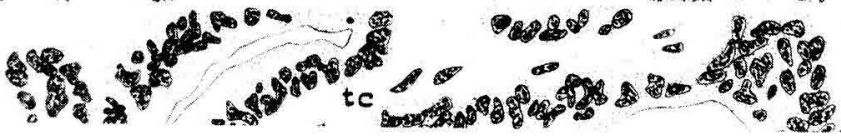

(3)
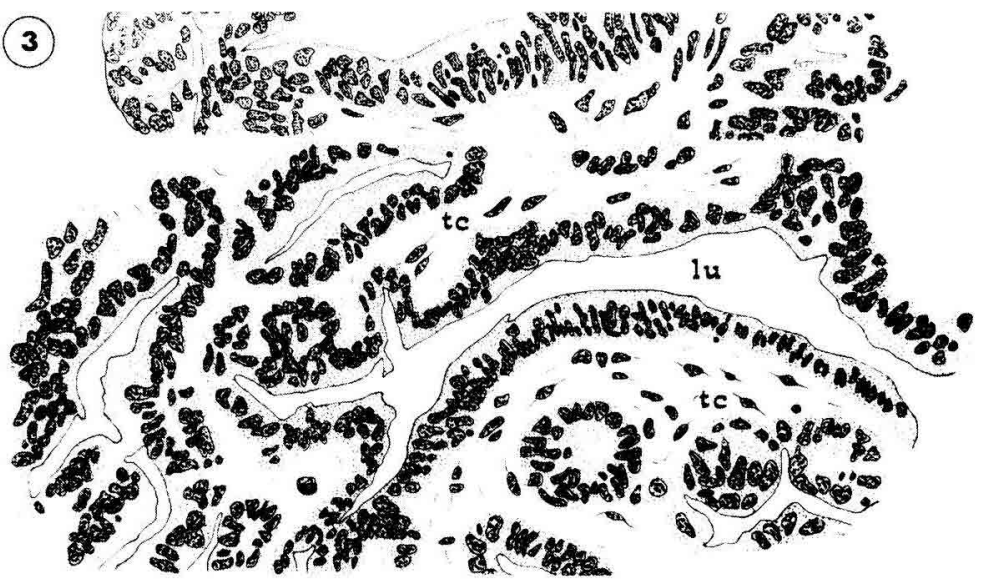


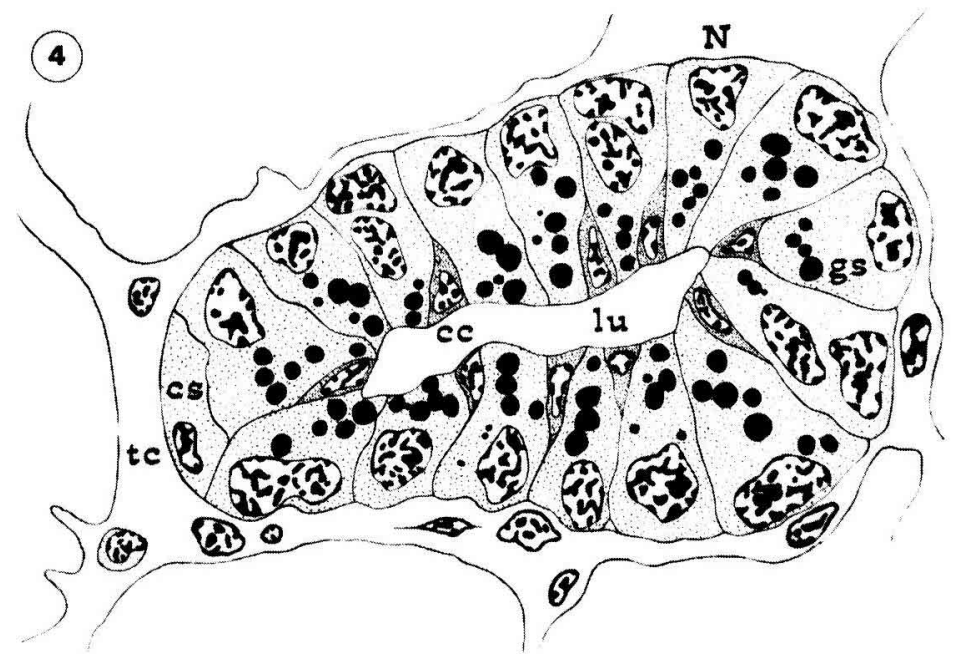

FIG. 4. - Coupe d'un acinus montrant l'apparition des premiers globules de sécrétion (gs) au pôle apical des cellules sécrétrices (cs). N = Noyau.

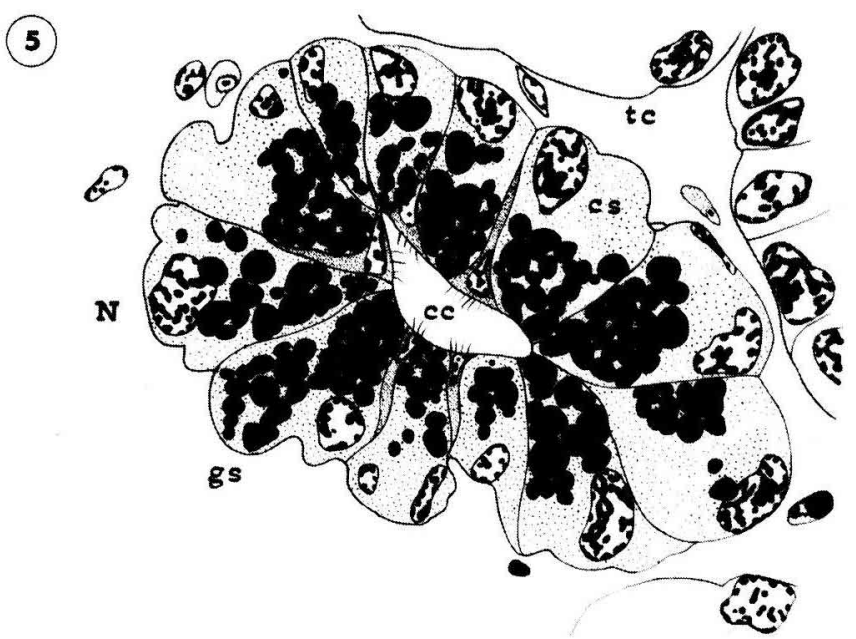

FIG. 5. - Acinus d'une glande à albumen entièrement différenciée avec des cellules ciliées (cc) et des cellules sécrétrices (cs) contenant des grains de sécrétion (gs) qui s'accumulent au pôle apical, puis remplissent toute la cellule. $\mathrm{N}$ : noyau d'une cellule sécrétrice ; tc : tissu conjonctif. 


\section{Etude ultrastructurale de la différenciation des cellules épithéliales.}

1) La cellule épithéliale indifférenciée avant la formation des premiers grains de sécrétion. - En microscopie électronique, les trois premiers stades morphogénétiques décrits (tubulaire simple, composé, acini) sont caractérisés par l'existence de cellules de type épithélial (fig. 1, PI. 1) qui constituent la paroi de la glande et la présence d'un tissu mésenchymateux en différenciation qui sépare les tubes.

Les cellules épithéliales indifférenciées ont une forme sensiblement cylindrique avec une extrémité appliquée contre la lame basale et l'autre délimitant une partie de la lumière des tubes (fig. 1, PI. I).

La face apicale, en regard de la lumière du tube ou de l'acinus, est hérissée de microvillosités tapissées d'un revêtement superficiel, la "fuzzy coat ». La face interne de la paroi cellulaire apicale est en étroite relation avec des microtubules et des microfilaments.

Les parois latérales comportent successivement, de la région apicale vers la partie basale :

- un desmosome de ceinture (" belt desmosome ") (fig. 2, PI. I) au niveau duquel l'espace intercellulaire est élargi, les membranes épaissies et le matériel adjacent dense. Cette formation correspond à une zone d'ancrage entre deux cellules voisines;

- des jonctions septées (« septate junctions »), type nid d'abeilles (fig. 2, PI. I), que l'on trouve fréquemment chez les Invertébrés. Les deux membranes plasmiques sont unies par des septes parallèles transverses qui sont en continuité avec la couche dense externe de la membrane. Ces jonctions occupent environ le tiers supérieur de la hauteur des cellules;

- des limites cellulaires classiques, qui occupent le reste de la paroi. On note la présence de quelques "gap junctions" (Staehelin, 1974). Par endroits (fig. 1, $\mathrm{Pl}$. I), I'espace intercellulaire est dilaté et contient probablement un liquide permettant les échanges ioniques entre les cellules.

La lame basale, bien constituée, épouse la face externe des cellules épithéliales ; elle forme une enveloppe qui présente des plis par endroits et émet alors des évaginations dans le tissu conjonctif adjacent. A sa surface, du côté des cellules épithéliales, on remarque des hémidesmosomes en relation avec des microfilaments et de petites vésicules.

Le noyau est très volumineux et pléomorphe (fig. 1, PI. I). L'hétérochromatine est répartie en mottes dans le nucléoplasme ; abondante, elle tapisse aussi la membrane interne de l'enveloppe nucléaire, à l'exception des zones où se trouvent les pores nucléaires. L'euchromatine se présente sous forme de petits granules et de filaments ténus. On observe un ou deux nucléoles par section, souvent séparés de I'hétérochromatine par un fin halo clair. La membrane nucléaire externe est toujours tapissée de ribosomes.

Dans le hyaloplasme, les ribosomes sont nombreux. Aux premiers stades, le réticulum endoplasmique est peu abondant (fig. 1, PI. I). A un stade plus tardif, l'ergastoplasme va se localiser surtout le long des membranes cellulaires latérales. 
L'appareil de Golgi est formé de quelques dictyosomes par cellule. II n'est pas rare d'observer à leur proximité des formations denses et des corps multivésiculaires (fig. 1, PI. I).

Les mitochondries, localisées aux pôles cellulaires, présentent souvent des étranglements.

Si les organites cellulaires sont peu développés dans les cellules épithéliales indifférenciées, par contre le glycogène est abondant dès les premiers stades. Révélé par la méthode de Thiéry et Rambourg (1974), il se présente sous forme de rosettes caractéristiques, c'est-à-dire de particules $\alpha$ et d'unités $\beta$ libres. Dans les plages de glycogène, apparaissent des globules lipidiques vers lesquels le polysaccharide semble former des travées rayonnantes.

2) Apparition des premiers grains de sécrétion. - Ce stade correspond à un stade transitoire pendant lequel on remarque, à côté des cellules épithéliales indifférenciées précédemment décrites, certaines cellules qui élaborent les premiers grains de sécrétion (PI. II). Le noyau de ces cellules passe d'une forme allongée à une forme ovale et vient se localiser dans la portion basale de la cellule. La polarité de la cellule sécrétrice est déterminée par la place du noyau, mais aussi par les globules de sécrétion qui sont apicaux. L'ergastoplasme et l'appareil de Golgi sont peu développés (fig. 2, PI. IV), mais les plages glycogène-lipides en position intermédiaire sont encore abondantes à proximité des premiers grains de sécrétion (PI. II).

3) Différenciation de deux types cellulaires. - A partir du stade où apparaissent les granules de sécrétion, on peut distinguer des cellules sécrétrices et des cellules ciliées (fig. 6 texte).

a) Les cellules non sécrétrices, ciliées à leur pôle apical bordant la lumière des tubes, ont un noyau allongé en position intermédiaire dans les glandes en début de phase sécrétoire. A ce stade, les mitochondries sont nombreuses aux deux pôles des cellules et on assiste à la formation des corpuscules basaux et des cils qui ont une structure classique. Pendant la différenciation des cils, les microvillosités de ces cellules disparaissent. Le noyau des cellules ciliées migre vers l'apex de la cellule au fur et à mesure de l'augmentation de volume des cellules sécrétrices qui se garnissent de grains de sécrétion. Des cellules ciliées comparables ont été également décrites dans la glande à albumen d'Helix pomatia adulte (Nieland et Goudsmit, 1969).

\section{PLANCHE}

FIG. 1. - Cellules de type épithélial constituant la paroi de l'ébauche glandulaire : le glycogène (G), sous forme de rosettes, est abondant, les organites cytoplasmiques ont l'aspect de ceux de cellules embryonnaires. (aG : appareil de Golgi ; Lu : lumière ; mf : microfilaments ; mt : microtubule). $\times 17000$.

FIG. 2. - Jonctions rencontrées dans la zone apicale : un « belt desmosome " (BD) entoure les cellules au voisinage de la lumière de la glande ; à la suite, on observe une jonction septée (" septate junction $"$ ) (SJ). $\times 88000$. 


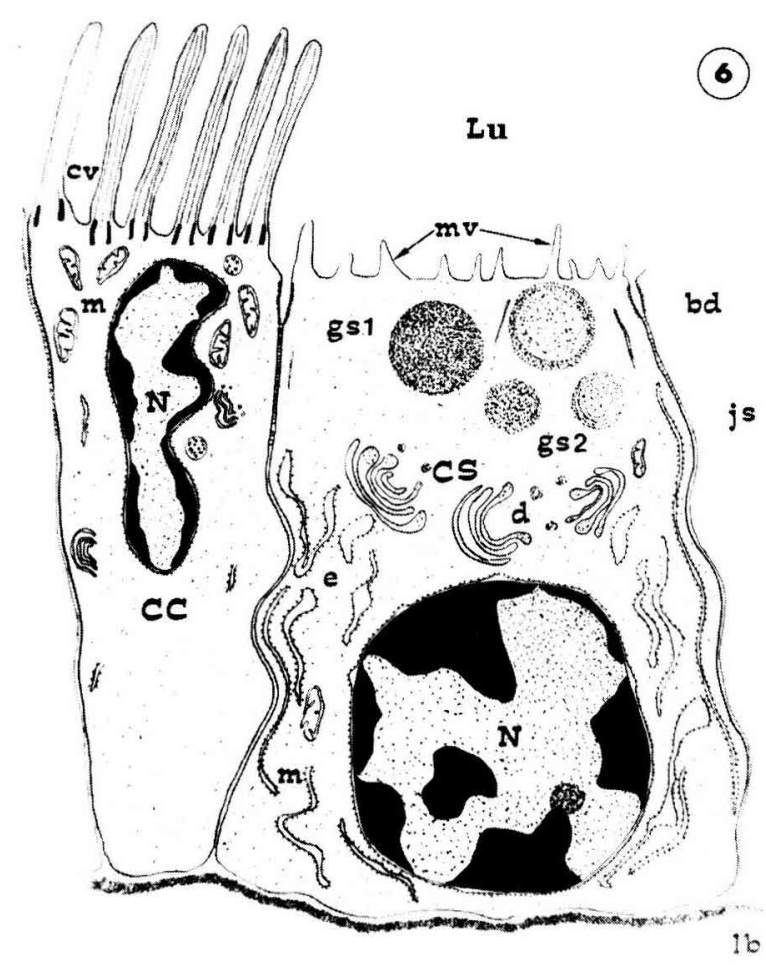

FIG. 6. - Schéma des deux types de cellules constituant les acini de la glande à albumen. bd : belt desmosome ; CC : cellule ciliée ; CS : cellule sécrétrice ; $c v$ : cil vibratile ; $d$ : dictyosome ; e : ergastoplasme ; gs1 et gs2 : grains de sécrétion ; js : jonction septée ; Lu : lumière de la glande ; $\mathrm{lb}$ : lame basale; $\mathrm{m}$ : mitochondries ; $\mathrm{mv}$ : microvillosités ; $\mathrm{N}$ : noyau.

\section{b) Les cellules sécrétrices (PI. III).}

a) Evolution des organites. La différenciation des cellules sécrétrices se fait progressivement.

Le noyau, de forme ovale, est volumineux et l'hétérochromatine apparaît plus homogène que dans les noyaux des cellules épithéliales indifférenciées. La membrane nucléaire externe est en étroite relation avec l'ergastoplasme.

\section{PLANCHE II}

Acini de glande à albumen au stade d'apparition de la sécrétion. Les noyaux (N) des cellules acineuses sont volumineux et riches en hétérochromatine. Certains d'entre eux passent d'une forme allongée à une forme ovale, correspondant à l'acquisition de leur position basale définitive : ce sont. les noyaux des cellules sécrétrices (il n'est pas possible à ce stade de distinguer les deux types cellulaires). Les premiers grains de sécrétion (GS) de 1,5 $\mu \mathrm{m}$ de diamètre ont un contenu homogène moyennement dense et ils apparaissent dans la région apicale. Les plages de glycogène (G) sont importantes, elles se répartissent entre les deux pôles de la cellule ( $L u$ : lumière ; lb : lame basale) et elles contiennent souvent des liposomes (L). $\times 6300$. 

Dans les cellules où apparaissent les premiers grains de sécrétion (PI. III et fig. 1, PI. IV), le réticulum granulaire est constitué de citernes aplaties et allongées parallèles aux parois latérales des cellules. Ensuite, l'ergastoplasme devient plus abondant; les citernes, plus nombreuses dans le hyaloplasme, possèdent un nombre beaucoup plus important de polysomes (fig. 3, PI. IV). Par endroits, I'ergastoplasme constitue des vésicules dilatées au contenu relativement dense aux électrons ou des citernes aplaties entourant des organites cellulaires (mitochondries, formations lipidiques).

Le complexe golgien, qui se présente à la fin de l'organogenèse sous forme d'un nombre réduit de saccules (fig. 2, PI. IV), se modifie et se développe. Le nombre de dictyosomes augmente. Les saccules, situés à proximité du noyau, allongés parallèlement à la paroi cellulaire au début de l'élaboration des grains (PI. III), se remplissent d'une substance dense aux électrons et bourgeonnent un nombre considérable de petites vésicules que l'on trouve à son voisinage parmi des vésicules de transition produites par le réticulum endoplasmique. Pendant la période de synthèse intense des grains de sécrétion, les dictyosomes prennent une forme courbée classique, les saccules golgiens contiennent un matériel granulaire polysaccharidique (technique selon Thiéry et Rambourg, 1974) et bourgeonnent des vésicules de grande taille (fig. 1, Pl. V) en forme de massues, qui se détachent et donnent les globules de prosécrétion.

ß) Les globules de sécrétion. Les premiers globules de sécrétion apparaissent un peu avant le développement important de l'ergastoplasme et des dictyosomes. A ce stade intermédiaire entre la fin de l'organogenèse et la phase de sécrétion généralisée, on note dans les cellules sécrétrices la juxtaposition de trois éléments importants (PI. III et fig. 1, PI. IV) : des globules lipidiques, de grandes plages glycogéniques et des globules de sécrétion dans la partie apicale des cellules.

A un stade plus avancé (sécrétoire généralisé), les globules de sécrétion se présentent sous différents aspects dans une même cellule. lls diffèrent entre eux par leur taille et par l'abondance de l'un ou l'autre de leurs constituants. Les composants polysaccharidiques Thiery positif sont soit uniformément répartis dans le globule (fig. 2 et $3, \mathrm{Pl}$. V), soit localisés à la périphérie (fig. $3, \mathrm{PI}$. V) ; ils sont quelquefois agencés en structure périodique. La composante protéique des grains est plus ou moins abondante et leur membrane est irrégulière.

Pendant la phase de formation intense des globules, les dictyosomes sont devenus très importants et se sont localisés dans la région supranucléaire. Les lipides disparaissent progressivement ainsi que le glycogène. Le mode de constitu-

\section{PLANCHE III}

Cellule sécrétrice en phase sécrétoire. La cellule élabore des globules (GS) plus denses dont la taille augmente de $1,3 \mu \mathrm{m}$ à $1,5 \mu \mathrm{m}$ de diamètre et certains atteignent la région des villosités apicales (vi) qui bordent la lumière (Lu). Les plages de glycogène (G) et les liposomes (L) sont en position intermédiaire. En même temps que l'on observe une évolution ultrastructurale des organites cellulaires (ergastoplasme, Golgi, mitochondries), les plages " glycogène-liposomes " disparaissent peu à peu. Le tissu conjonctif (TC) intertubulaire lâche des stades précédents est laminé entre les acini limités par la lame basale (lb). 


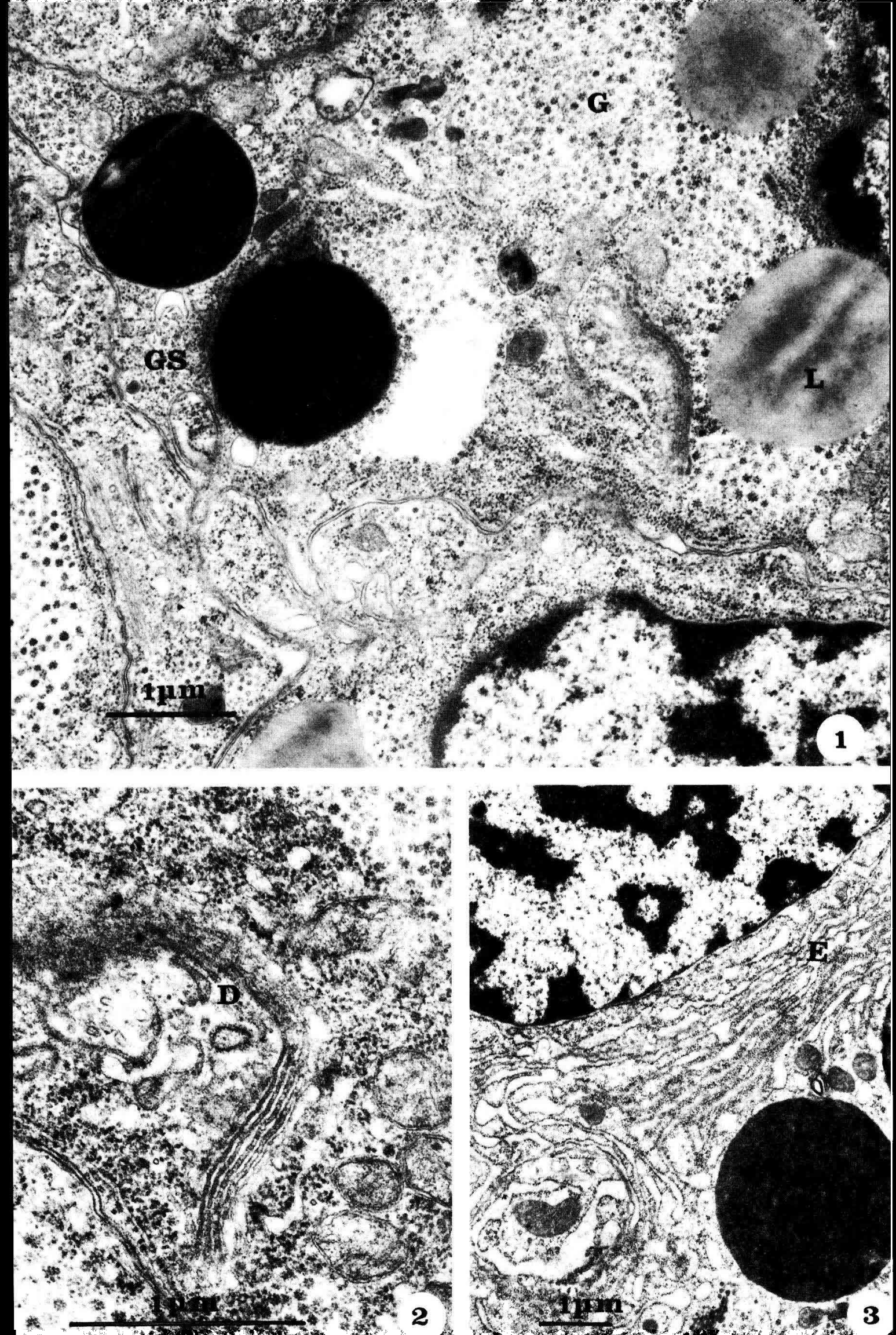


tion des globules de sécrétion semble plus homogène et se fait principalement à partir de grosses vésicules (fig. 1, Pl. V) en forme de massue résultant du bourgeonnement des saccules golgiens. La polarité de la cellule se caractérise alors par son noyau basal, ses dictyosomes en position supranucléaire et ses globules de sécrétion apicaux (proches des villosités) qui vont progressivement envahir toute la cellule et refouler le noyau contre la lame basale.

\section{Discussion.}

La discussion des résultats porte sur deux aspects originaux mis en évidence au cours de cette étude. Le premier concerne l'évolution de l'architecture de la glande à albumen. Le deuxième se rapporte à la différenciation des cellules épithéliales en cellules sécrétrices en fonction des connaissances actuelles de la biologie cellulaire.

\section{1) Caractères particuliers de la morphogenèse.}

La glande à albumen apparaît comme un diverticule de l'épithélium palissadique du tractus génital au niveau de la jonction du canal hermaphrodite et du spermoviducte. Jusqu'à présent, l'évolution de la glande à albumen avait surtout été décrite chez les Arionidés et les Limacidés (Laviolette, 1954 ; Lusis, 1961 ; Smith, 1966 ; Runham et Laryea, 1968) en fonction des phases de croissance du tractus génital. Chez Helix aspersa, les recherches effectuées sur la différenciation du tractus génital (Courtot, 1975, 1977 ; Enée et al., 1977) montrent que la glande à albumen acquiert sa structure acineuse chez des animaux de 3 à 5 mois avant qu'apparaissent les premiers grains de sécrétion, tandis que la prostate, dont les cellules se différencient également à partir de cellules épithéliales indifférenciées d'une invagination longitudinale du spermoviducte, présente une activité sécrétrice beaucoup plus précoce ( 3 mois) dès le bourgeonnement des premiers tubules prostatiques. Ces observations nous amènent à considérer que la différenciation des organes annexes du tractus génital à partir du conduit épithélial primaire, se fait sous l'influence "d'inducteurs" régionaux comparables aux interactions tissulaires épithélio-mésenchymateuses mises en évidence chez les Vertébrés. En effet, on note qu'au stade de formation des acini, l'interpénétration entre les cellules épithéliales et le conjonctif est particulièrement importante par la formation de digitations de la base des cellules épithéliales et le plissement de la lame basale. Une telle configuration a également été signalée par Boisseau (1973) pendant la différenciation des cellules épithéliales de l'oviducte du Pleurodèle et

PLANCHE IV

FIG. 1. - Stade intermédiaire: coexistence des globules lipidiques (L), des plages de glycogène (G) et des globules de sécrétion (GS) dans une même cellule. $\times 22500$.

FIG. 2. - Dictyosome (D) à activité relativement faible (étape d'apparition de la sécrétion). $\times 27000$.

FIG. 3. - L'ergastoplasme (E) se déve/oppe et se présente sous forme de longues citernes circonvoluant dans le hyaloplasme. Les dicytosomes deviennent plus nombreux. $\times 14300$. 
l'auteur « soupçonne un rôle inducteur du tissu conjonctif dans la différenciation des cellules épithéliales ». Dans le cas de la glande à albumen, comme dans le cas de la différenciation de l'oviducte des Vertébrés, ce phénomène d'induction dépend de facteurs endocrines. Mais, tandis que le rôle des hormones sexuelles a été bien démontré chez les Vertébrés (Boisseau, 1973; Sandoz et BoisvieuxUlrich, 1976), chez les Gastéropodes Pulmonés, les connaissances sont moins précises. Cependant, l'influence de la gonade sur la croissance et l'activité de la glande à albumen de Limaces a été démontrée par des expériences de castration (Laviolette, 1954) et l'action du cerveau sur l'activité sécrétoire des cellules glandulaires de la glande à albumen d'Helix pomatia a été mise en évidence en culture in vitro par Goudsmit (1975). Les corps dorsaux accolés aux ganglions cérébraux agiraient également sur la différenciation des organes sexuels accessoires femelles chez Lymnaea stagnalis (Geraerts et Joosse, 1975 ; Geraerts et Algera, 1976) et chez Agriolimax reticulatus (Wijdenes et Runham, 1976). Les premiers essais d'étude expérimentale réalisés en culture in vitro (Courtot, 1977 ; Gomot et Courtot, 1979) montrent que pendant la phase d'organogenèse, les ganglions cérébraux ont une action directe et induisent une multiplication des cellules. A ce stade, la glande hermaphrodite juvénile et adulte est sans effet, tandis que les tentacules oculaires sont inhibiteurs.

\section{2) Modalités de la différenciation glandulaire et de la sécrétion.}

Au cours de la différenciation des cellules sécrétrices, on constate que l'ergastoplasme, puis l'appareil de Golgi, prennent une importance croissante et que les réserves polysaccharidiques sous forme de rosettes et les lipides diminuent. Cette augmentation de l'activité élaboratrice de l'ergastoplasme et de l'appareil de Golgi est commune à de nombreux organes de Vertébrés, bien que la composition des grains de sécrétion soit différente suivant l'organe considéré. Des modifications comparables de l'ergastoplasme et de l'appareil de Golgi ont été décrites pendant la différenciation naturelle ou artificielle d'organes comme le

\section{PLANCHE V}

FIG. 1. - Organites cellulaires d'une cellule sécrétrice à un stade avancé. L'ergastoplasme (E) est dilaté, les dictyosomes (D) sont courbés et bourgeonnent des vésicules en forme de massues qui confluent en donnant les globules de prosécrétion (GP). On note la présence de matériel granulaire abondant dans les dictyosomes. $\times 29000$.

FIG. 2. - Mise en évidence des polysaccharides d'après la technique de Thiéry et Rambourg (1974) : la préparation révèle la nature polysaccharidique du constituant granulaire du grain de sécrétion. $\times 55000$.

FIG. 3. - Divers types de globules dans la même cellule : - globules de sécrétion clairs à deux constituants (GS I) : le matériel granulaire est réparti de façon homogène ; - globules de sécrétion foncés (GS II) où le matériel granulaire forme une couche régulière à la périphérie. Les globules GS II ne semblent pas posséder de membrane. $\times 82000$.

FIG. 4. - Le globule de sécrétion est acheminé vers l'apex de la cellule grâce à un système de microtubules (mt). $\times 55000$. 
pancréas (Kalmann et Grobstein, 1964), l'oviducte de Poule (Palmiter et Wrenn, 1971 ; Kellokumpu-Lehtinen et al., 1976), les glandes sous maxillaires de Souris (Chrétien, 1972), I'oviducte de Pleurodèle (Boisseau, 1973).

Dans le cas de la glande à albumen, on observe des changements dans la structure des grains au fur et à mesure de la différenciation glandulaire des cellules. Ces changements correspondent probablement à des modifications qualitatives des synthèses cellulaires. En effet, les premiers grains de sécrétion apparaissent dans des cellules dont les organites classiques de synthèse (ergastoplasme et appareil de Golgil sont peu développés, alors que les plages de glycogène et les globules lipidiques sont abondants. II est possible que les réserves de glycogène soient " recyclées » et fournissent au moins une partie des oses constituants des glycoprotéines granulaires des premiers grains de sécrétion. Les grains qui se forment ensuite seraient le résultat du mode de synthèse classique faisant intervenir l'ergastoplasme pour la synthèse des protéines et l'appareil de Golgi pour la formation des glycoprotéines. L'existence de plusieurs types de grains de sécrétion au cours de l'ontogenèse a été signalée dans la glande sous mandibulaire du Rat (Denny et Cope, 1977). Mais il arrive également que la même cellule présente des grains de structure différente comme cela a été observé dans les glandes des sinus maxillaires de l'Homme (Vidic et Tandler, 1977) ; dans ce cas, on peut supposer que les différences d'aspect peuvent être dues à une séquence de maturation des grains. En ce qui concerne la glande à albumen, les différences d'aspect des grains correspondent peut-être à des degrés de concentration de la substance fondamentale protéique décrite dans les grains de sécrétion de la glande à albumen d'Helix pomatia adulte (Nieland et Goudsmit, 1969) ou à une différence de nature des polysaccharides car Goudsmit $(1964,1975)$ a montré par dosage biochimique que la glande sécrète du glycogène et du galactogène et que les proportions de ces deux constituants varient suivant l'état physiologique chez Helix pomatia. L'examen ultrastructural des grains de sécrétion ne permet pas de savoir si ces deux polysaccharides sont présents dans les mêmes grains ou s'ils sont contenus dans des grains différents. Une analyse autoradiographique fine couplée avec des digestions enzymatiques devra être entreprise pour répondre à cette hypothèse. On doit également signaler que Nieland et Goudsmit (1969) n'avaient pas observé de membrane autour des grains de sécrétion de la glande adulte d'Helix pomatia, tandis que dans les glandes d'Helix aspersa, au début de l'entrée en fonction, on note des grains sans membrane et des grains avec membrane, ce qui traduit peut-être deux modes de formation. Enfin, dans les stades étudiés, nous avons régulièrement observé l'existence de microtubules parallèles aux parois latérales des cellules sécrétrices dans la zone de formation des grains et dans la zone apicale d'accumulation (fig. 4, PI. V), comme dans les cellules du pancréas exocrine et d'autres types cellulaires suggérant que ces formations peuvent intervenir dans le cheminement des grains et dans leur décharge (Palade, 1975).

En conclusion, la glande à albumen apparaît comme un modèle intéressant de différenciation cellulaire chez les Invertébrés. Nous avons décrit les principaux aspects cytologiques de la glande au cours de son organogenèse et de son entrée en fonction et les premiers résultats expérimentaux obtenus en culture in vitro 
nous indiquent que contrairement à ce qui se passe pour le tractus génital des Vertébrés, ce sont plutôt les sécrétions nerveuses que les sécrétions gonadiques qui agissent sur la différenciation des organes annexes du tractus génital. Ces résultats doivent être complétés par d'autres expérimentations et des études biochimiques afin de préciser le mode d'action des facteurs de différenciation sur les cellules cibles du tractus génital.

Reçu en novembre 1981. Accepté en mars 1982.

Remerciements. - Les auteurs remercient Claude Colard, Brigitte Jolibois et Alfred Martin pour leur aide dans la réalisation du manuscrit de cette publication.

\section{Références}

ABELOOS M., 1943. Effets de la castration chez un Mollusque Limax maximus. C.R. Acad. Sci., Paris, 216, 90-92.

BOISSEAU C., 1973. Etude ultrastructurale de l'oviducte du Triton Pleurodeles waltiii Michah. II. Morphogenèse des glandes et différenciation des cellules épithéliales de l'oviducte moyen. J. Microscopie, 18, 359-382.

BOLOGNANI FANTIN A. M., GERVASO M. V., 1971. A histoenzymatic investigation of galactogen synthesis in the albumen gland of Helix pomatia and Lymnaea stagnalis. Histochemistry, 28, 88-94.

BRACHET J., 1944. Embryologie chimique. Desoer, Liège.

CHRÉTIEN M., 1972. Action de la testostérone sur la structure fine d'un effecteur : la glande sousmaxillaire de la Souris mâle. II. Réaction des tubes sécréteurs à l'injection de testostérone chez le castrat. J. Microscopie, 14, 55-74.

COURTOT A. M., 1975. Contribution à l'étude de l'organogenèse et de l'activité de la glande à albumen chez Helix aspersa Müll. (Pulmoné Stylommatophore). Ann. sci. Univ. Besançon, Zool. Physiol. Biol. anim., 12, 99-133.

COURTOT A. M., 1977. Étude cytologique et expérimentale de la differenciation et de la sécrétion de la glande à albumine de l'Escargot: Helix aspersa Müll. Th. $3^{\circ}$ cycle, Besançon.

DE JONG-BRINK M., 1969. Histochemical and electron microscope observation on the reproductive tract of Biomphalaria glabrata (Australorbis glabratus) intermediate host of Schistosoma mansoni. Z. Zellforsch., 102, 507-542.

DENNY P. C., COPE P., 1977. Age-specific differentiations during the development of the rat submandibular gland. Differentiation, 8, 105-111.

ENEE J., 1980. Contribution à l'étude de la croissance de l'Escargot Helix aspersa Müller. Ontogenèse de l'appareil génital et recherche cytologique sur la différentiation et la sécrétion de la prostate. Th. $3^{\mathrm{e}}$ cycle, Besançon.

ENEE J., GOMOT L., BRIDE M., 1977. Différenciation de la prostate chez l'Escargot Helix aspersa Müll. C.R. Soc. Biol., 171, 728-732.

GERAERTS W. P. M., ALGERA L. H., 1976. The stimulating effect of the dorsal body hormone on cell differentiation in the female accessory sex organs of the hermaphrodite freshwater snail, Lymnaea stagnalis. Gen. comp. Endocr., 29, 109-118.

GERAERTS W. P. M., JOOSSE J., 1975. Control of vitellogenesis and growth of female accessory sex organs by the dorsal body hormone DBH in the hermaphroditic freshwater snail Lymnaea stagnalis. Gen. comp. Endocr., 27, 450-464.

GOMOT L., COURTOT A. M., 1979. Étude en culture in vitro du contrôle endocrine de la glande à albumen chez l'Escargot Helix aspersa. Malacologia, 18, 361-367.

GOMOT L., ENEE J., 1980 . Biologie de la reproduction de l'Escargot Helix aspersa Müll. : les phases de croissance et la différenciation sexuelle. Atti Acad. Fisiocritici Siena, 73-85. 
GOUDSMIT E. M., 1964. The metabolism of galactogen and glycogen by the pulmonata snails Bulimnaea megasoma and Helix pomatia. Diss. Abstr., 25, 37-61.

GOUDSMIT E. M., 1975. Neurosecretory stimulation of galactogen synthesis within the Helix pomatia albumen gland during organ culture. J. exp. Zool., 191, 193-197.

GOUDSMIT E. M., ASHWELL G., 1965. Enzymatic synthesis of galactogen in the snail Helix pomatia. Biochem. biophys. Res. Commun., 19, 417-422.

GOUDSMIT E. M., FRIEDMAN T. B., 1976. Enzymatic synthesis and interconversion of UDPglucose and UDP-D-galactose in the albumen gland of the snail Helix pomatia. Comp. Biochem. Physiol., 54B, 135-139.

GOUDSMIT E. M., NEUFELD E. F., 1967. Formation of GDP-L-galactose from GDP-D-manose. Biochem. biophys. Res. Commun, 26, 730-735.

KALMANN F., GROBSTEIN C., 1964. Fine structure of differentiation mouse pancreatic exocrine cells in transfilter culture. J. Cell Biol., 20, 399-413.

KELLOKUMPU-LEHTINEN P., JOKELAINEN P. T., TUOHIMAA P., 1976. Early cytodifferentiation of the chick oviduct epithelium by progesterone. J. u/trastruct. Res., 54, 190-201.

LAVIOLETTE P., 1954. Rôle de la gonade dans le déterminisme humoral de la maturité glandulaire du tractus génital chez les Gastéropodes Arionidae et Limacidae. Bull. Biol., 86, 310-322.

LISON L., 1960. Histochimie et cytochimie animales. Principes et méthodes. 2 vol., GauthierVillars Ed., Paris.

LUFT J. H., 1961. Improvements in epoxy resin embedding methods. J. biophys. biochem. Cytol., 9, 409-414.

LUSIS O., 1961. Post embryonic changes in the reproductive system of the slug Arion rufus. Proc. zool. Soc. Lond, 137, 433-468.

McGEE-RUSSEL S. M., SMALE N. B., 1963. On colouring epon embedded tissue sections with Sudan black B or nile blue A for light microscopy. Quart. J. micr. Sc., 104, 109-115.

MEENAKSHI V. R., SCHEER B. T., 1969. Regulation of galactogen synthesis in the slug Agriolimax columbianus. Comp. Biochem. Physiol., 29, 119-140.

NIELAND M. L., GOUDSMIT E. M., 1969. Ultrastructure of galactogen in the albumen gland of Helix pomatia. J. ultrastruct. Res., 29, 119-140.

PALADE G., 1975. Intracellular aspects of the process of protein secretion. Science, 189, 347-358.

PALMITER R. D., WRENN J. T., 1971. Interaction of oestrogen and progesterone in chick oviduct development. III. Tubular gland cell differentiation. J. Cell. Biol., 50, 593-615.

REYNOLDS E. S., 1963. The use of lead citrate at high $\mathrm{pH}$ as an electrron opaque stain in electron microscopy. J. Cell Biol., 17, 208-212.

RUNHAM N. W., LARYEA A. A., 1968. Studies on the maturation of the reproductive system of Agriolimax reticulatus (Pulmonata : Limacidae). Malacologia, 7, 93-108.

SANDOZ D., BOISVIEUX-ULRICH E., 1976. Ciliogenèse dans les cellules à mucus de l'oviducte de Caille. I. Étude ultrastructurale chez la Caille en ponte. J. Cell Biol., 71, 449-459.

SAWICKA T., 1967. Glucose 1-phosphate uridyl transferase of Helix pomatia. Bull. Acad. pol. Sci., II, 521-525.

SMITH B. J., 1966. Maturation of the reproductive tract of Arion ater (Pulmonata Arionidae). Malacologia, 4, 325-349.

SPÜRR A. R., 1969. A low viscosity epoxy resin embedding medium for electron microscopy. J. ultrastruct. Res., 26, 31-43.

STA'EHELIN L. A., 1974. Structure and function of intercellular junctions. Intern. Rev. Cytol., 39, 191-283.

THIÉRY J. P., RAMBOURG A., 1974. Cytochimie des polysaccharides. J. Microscopie, 21, 225-232.

TRUMP B. F., SMUCKLER E. A., BENDITT E. P., 1961. A method for staining epoxy sections for light microscopy. J. ultrastruct. Res., 5, 343-345.

VIDIC B., TANDLER B., 1977. Ultrastructure of the secretory cells of the submucosal glands in the human maxillary sinus. J. Morph., 150, 167-182.

WIJDENES J., RUNHAM N. W., 1976. Studies on the function of the dorsal bodies of Agriolimax reticulatus (Mollusca: Pulmonata). Gen. comp. Endocr., 29, 545-551. 